Mom ̌̇ilo D. Savić

\title{
IL CONDIZIONALE TEMPORALE NELLE TRE REDAZIONI DEL ROMANZO MANZONIANO
}

E noto che l'italiano, a differenza delle altre lingue neolatine occidentali, possiede due tempi per esprimere il futuro in relazione al passato: non soltanto il condizionale semplice, ma anche quello composto. ${ }^{1}$ Essendo il condizionale semplice un tempo che di secolo in secolo sta perdenoio terreno in quest'uso (e lo può dimostrare l'impiego più che parco che se ne fa nelle opere letterarie moderne, ${ }^{2}$ nonché qualche manuale grammaticale ${ }^{3}$, crediamo utile analizzare tutti $i$ condizionali temporali (trascurando assolutamente quelli con funzione modale) nelle tre redazioni successive del romanza manzoniano; tanto più che il Manzoni è uno scrittore che si proponeva di avvicinare la lingua letteraria a quella parlata ${ }^{4}$ e che molte volte anche l'italiano moderno, volendo darsi l'approvazione di qualche costrutto sintattico, si richiama ai Promessi Sposi.

Ritenendo che potrebbe risultare assai indicativo l'uso di dati statistici a conferma di certì risultati di carattere strettamente filologico, ci siamo decisi a seguire un metodo finora poco praticato. Con ciò non intendiamo esaurito il problema. Promettendo di tornare su di esso un'altra volta, ora crediamo che il largo uso di specchietti statistici di cui ci siamo valsi possa riuscire utile ad una visione più chiara, sebbene parziale, dell'intero problema e che i risultati numerici comportino conclusioni, se non decisive, interessanti.

In base alle richerche statistiche che abbiamo fatto nelle tre dette redazioni $^{5}$ possiamo rappresentare l'uso dei condizionali temporali con la seguente tabella:

${ }^{1}$ Ricorrendo a una terminologia poco usata, cioè aI condizionale usemplicer e al wcompostou invece del "presenter e del "passatou, sottolineiamo che c'interessiamo solo della loro applicazione temporale (cioè come futuro in relazione al passato) e non di quella modale. - Dobbiamo aggiungere che non abbiamo tenuto conto d'altre possibilità che possano servire ad esprimere la stessa relazione.

${ }^{2}$ P. e., nel romanżo Mille tradimenti di Ugo Pirro, Milano, 1959, nelle 350 pagine non esiste nessun condizionale semplice che funga da futuro in relazione al passato; quindi il composto vi è generalizzato.

${ }^{3}$ P. e., Giacomo Devoto, Introduzione alla grammatica, Firenze, 1941, p. 140, vede il mezzo per esprimere questa relazione solo nel condizionale composto.

${ }^{4}$ Cfr. Bruno Migliorini, Lingua e cultura, Roma', 1948, p. 31.

${ }^{5}$ Nelle nostre ricerche ci siamo serviti delle tre redazioni del romanzo manzoniano in: Tutte le opere di Alessandro Manzoni, vol. II, tomo III - Fermo $e$ Lucia (1821-1823) - segnato $a$; tomo II - I Promessi Sposi (1825-1827) - segnato b; tomo I - I Promessi Sposi (1840) - segnato c, Milano, 1959. 


\begin{tabular}{|c|c|c|c|c|c|c|c|c|c|}
\hline Redazione & $\begin{array}{c}\text { Condizio- } \\
\text { nale }\end{array}$ & 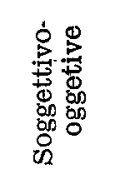 & 蛋 & 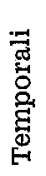 & 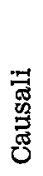 & 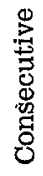 & 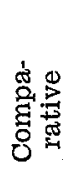 & 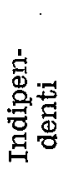 & 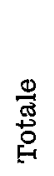 \\
\hline \multirow{2}{*}{$\begin{array}{l}\text { Fermo e Eucia } \\
\quad(1821-1823)\end{array}$} & semplice & $49(7)$ & 9 & 4 & - & - & - & 2 & 64 \\
\hline & composto & $108(16)$ & 20 & 2 & - & - & - & 1 & 131 \\
\hline \multirow{2}{*}{$\begin{array}{l}\text { I Promessi } \\
\text { Sposi } \\
\text { (1825-1827) }\end{array}$} & semplice & $67(4)$ & 7 & 3 & 1 & - & - & 10 & 88 \\
\hline & composto & 14 & 7 & 3 & - & 1 & - & 2 & 27 \\
\hline \multirow{2}{*}{$\begin{array}{l}\text { I Promessi } \\
\text { Sposi }(1840)\end{array}$} & semplice & $63(9)$ & 16 & 2 & - & - & 1 & 9 & 91 \\
\hline & composto & $33(2)$ & 6 & - & - & - & 一 & 1 & 40 \\
\hline
\end{tabular}

I numeri fra parentesi nella colonna usoggetivo-oggetive" indicano quante volte nel numero precedente la soggettivo-oggettiva è condizionata da una protasi ovvero dall'effettuarsi di un'altra azione, sia all'indicativo: ». .. e si sarrebe trovato che se uno meritava la galea l'altro avrebbe dovuto andare a fargli compagnia:" (a, I, IV, p.62); sia al congiuntivo: ", gli fecero promettere che... se potesse, verrebbe a desinare con loro." ( $c$, XXXVIII, p. 658); sia al condizionale: "La buona donna le promise che appena suo marito tornerebbe dalla Chiesa, ella lo, determinerebbe ad andare in cerca, $(a$, III, II, p. 357); ossia con una forma implicita: »;e ch'egli sperava che colui, non mi veggendo, non si curerebbe piu di me.« (c, III, p. 41). Comunque, in tutti questi casi si tratta di preteriti del futuro in relazione al passato. che condizionano l'avverarsi dell'azione posteriore.

Parlando del romanzo manzoniano si accenna di solito alla differenza che esiste tra la prima e la seconda redazione (sono stati infatti pubblicati interi volumi che comprendono episodi che non si trovano nel secondo e nell'ultimo rifacimento ${ }^{6}$ ), mentre si ritiene che l'edizione del 1840 sia soltanto una assai limitata rielaborazione di quella precedente. Riguardo al problema, invece, che si siamo posti, si addiviene a certe conclusioni ben diverse: la nostra tabella c'insegna che il Manzoni, dopo aver introdotto tanti condizionali composti nella prima redazione, ne ha diminuito il numero al minimo nella seconda per riaumentarlo discretamente in quella definitiva.

Questa nostra affermazione la renderemo più chiara presentando parte della tabella precedente in \% (tedendo conto soltanto delle soggettivo-oggettive e del numero totàle):

- Facciamo menzione solo dei Brani inediti dei Promessi sposi di Alessandro Manzoni, per cura di Giovanni Sforza, Milano, 1905. 


\begin{tabular}{|c|c|c|c|}
\hline Redazione & Condizionale & $\begin{array}{c}\text { Soggettivo- } \\
\text { oggetive }\end{array}$ & $\begin{array}{c}\text { Totale } \\
\text { (comprese le indi- } \\
\text { pendenti e tutte } \\
\text { 1e dipendenti) }\end{array}$ \\
\hline $\begin{array}{c}\text { Fermo e Lucia } \\
(1821-1823)\end{array}$ & semplice & 31,21 & 32,82 \\
\hline $\begin{array}{c}\text { composto } \\
\text { I Promessi Sposi }\end{array}$ & semplice & 68,79 & 67,18 \\
\hline I Promessi Sposi & composto & 82,72 & 76,52 \\
\hline \begin{tabular}{l} 
(1840) \\
\hline
\end{tabular} & semplice & 17,28 & 23,48 \\
\hline
\end{tabular}

Dopo esaminate queste percentuali, possiamo porci una domanda: è riuscito il Manzoni ad avvicinare la lingua letteraria a quella parlata anche riguardo al problema di cui c'interessiamo?

Prima di dare una risposta decisiva dobbiamo rivolgere lo sguardo ai mezzi esprimenti la relazione di cui ci occupiamo nei periodi che precedono la lingua letteraria del primo Ottocento. Non tenendo conto degli altri mezzi che servono ad esprimere questa o una simile relazione fin dal periodo che precede la formazione della lingua italiana e di cui certi di poca importanza continuano a vivere tuttora, ${ }^{7}$ possiamo confermare - in base a un gran numero di testi di tutti i secoli della letteratura italiana che abbiamo avuto in mano - che il condizionale semplice comincia ad apparire come futuro in relazione al passato appena dal Duecento, assumendo un significato temporale invece di quello modale di cui era esclusivamente rivestito prima. Quanto al condizionale composto nella stessa funzione, esso viene a riaffermarsi qualche secolo più tardi: pur trovandolo nel Trecento (Compagni, Villani), non dobbiamo attribuire troppa importanza a questo fatto, essendo sospetti questi testi di aver subito forti influssi da parte della sintassi quattrocentesca. Il Quatrocento però ci dà esempi sicuri di condizionale composto temporale. Sebbene usato abbastanza parcamente, lo vediamo apparire su una vasta area che si stende da Venezia fino a Salerno (Marin Sanudo, San Bernardino da Siena, Masuccio Salernitano). Nel secolo successivo, non essendo più una rarità, comincia ad acquistar terreno a spese del condizionale semplice, e lo troviamo nelle opere di un gran numero di scrittori di diversa cultura (Cellini, Varchi, Bandello, Vasari, Di Costanzo, Caro). Si ha l'impressione che il processo di sostituzione del condizionale semplice con

${ }^{7}$ Accennando ad altre possibilità che soddisfino questa relazione - sistematicamente esposte per i tempi più remoti delle lingue neolatione da parte di Gerhard Rohlfs, Das romanische shabeow-Futurum und Konditionalis (Archivum Romanicum VI, 1, 1922, p. 146) - fermiamoci solo ad una, cioè all'imperfetto del verbo dovere + infinitivo, tanto più che questa perifrasi appare anche nella lingua moderna: "disse privato alli soi ca fra iij dii dovea ritornare allo pavillione." (Storie de Troia e de Roma, p. 383 in La prosa del Duecento, a cura di Cesare Segre e Mario Marti, Milano-Napoli, 1959). 
quello composto sia andato svolgendosi in maniera particolarmente intensa nel tardo Cinquecento e nel Seicento che, rivelando un gusto per l'ampollosità, ha ridotto il condizionale semplice temporale al minimo. Questo processo secondo la mia opinione - si concluse nel Settecento, almeno per quel che riguarda la lingua parlata, mentre gli scrittori, seguendo la ricca tradizione letteraria, continuavano a adoperare, sebbene di rado, anche il condizionale semplice temporale.

Non potendo trattenerci qui sull'essenza di questo fenomeno che esige ampie discussioni, affermiamo solo che questa sostituzione ha avuto luogo in seguito ad uno spostamento dei tempi avveratosi nel periodo storico della lingua italiana.

Ritornando al romanzo manzoniano, possiamo aggiungere che gli scrittori che si affermano successivamente all' epoca manzoniana (Nievo, Fogazzaro, Verga) s'attengono all'uso del condizionale temporale che ne fanno gli autori del Settecento (Goldoni, Carlo Gozzi), e dobbiamo dunque concludere che il Manzoni e una buona parte dei suoi contemporanei (Foscolo, Pellico, Guerrazzi) non seguivano l'uso della lingua viva, ma si proponevano - quanto al condizionale temporale - di continuare la tradizione letteraria italiana. Analizzando le percentuali in cui appaiono i condizionali temporali, veniamo alla conclusione che il Manzoni, dopo aver seguito prevalentemente la lingua viva nella prima redazione del suo romanzo; si è lasciato influenzare dalla tradizione letteraria nella seconda, dove la percentuale dei condizionali composti temporali ammonta appena a 17,28 per le soggettivo-oggettive, ossia a $23,4 \mathrm{~s}$ nel totale. Nell'ultima redazione, egli tornò ad aumentare - sebbene questo non sia evidente a prima vista (senza un'analisi più sottile) - la percentuale della forma viva, pur restando fedele alla tradizione ietteraria e allontanandosi dal linguaggio quotidiano.

Parlando dei condizionali temporali nel romanzó manzoniano ci siamo occupati finora soltanto delle soggettivo-oggettive. Per le altre dipendenti, il senso temporale viene dato da qualche locuzione avverbiale o dall'intero contesto, p. e.: ":pensava che quando sarei sua moglie colui non ardirebbe più tormentarmi;" ( $a$, I, III, p. 45); "Parlò... che appena l'età lo avrebbe concesso, ella sarebbe assunta alla prima dignità; « ( $b, \mathrm{X}, \mathrm{p} .166)$; »,faceva loro scontare anticipatamente $i$ piaceri che avrebbero goduti un giorno." $(c, \mathrm{X}$, p. 185); »le fece accettare un gruppetto di scudi, per riparare al guasto che troverebbe in casa; " (c, XXX, p. 521).

L'uso del condizionale temporale nelle indipendenti si spiega invece col discorso indiretto libero. Sebbene questa innovazione sia stata constatata. appena dall'epoca postmanzoniana, ${ }^{8}$ noi siamo sicuri che la possiamo seguire sporadicamente dai tempi più remoti della letteratura italiana.? Di conse-

${ }^{8}$ Cfr. Nicola Vita, Genesi del "discorso rivissuto« e suo uso nella narrativa. italiana in Cultura neolatina XV, 1-2, 1954,pp. 5-34. [Si veda ora anche G. Herczeg, Lo stile indiretto libero; Firenze, 1963. - N. d. R.]

${ }^{9}$ Cito solo un esempio del Sacchetti, credendo che se ne possano trovare di più antichi: "il pregò per amor di Dio che si crollasse nel pozzo e togtiesse un buon 
guenza, gli esempi di questo tipo sono ad attribuire al discorso indiretto libero: ":quanto alle domande espresse, avrebbe provveduto nel miglior moăo che il tempo e le necessità avessero conceduto." (b, XXXII, p. 547); ";e lui prendeva la cura di tutto il resto, e guiderebbe la cosa.« (c, XX, p. 343).

Quanto all'uso dei due condizionali temporali differenti nello stesso periodo, dobbiamo dire che questi casi si trovano di rado nel romanzo manzoniano, e lo possiamo presentare con la seguente tabella:

\begin{tabular}{|c|c|c|}
\hline Redazione & $\begin{array}{c}\text { Condizionale sem- } \\
\text { plice + condizio- } \\
\text { nale composto }\end{array}$ & $\begin{array}{c}\text { Condizionale com- } \\
\text { posto + condizio- } \\
\text { nale semplice }\end{array}$ \\
\hline $\begin{array}{c}\text { Fermo e Lucia } \\
(1821-1823)\end{array}$ & 1 & $3(1)$ \\
\hline $\begin{array}{c}\text { I Promessi Sposi } \\
(1825-1827)\end{array}$ & $1(1)$ & $3(1)$ \\
\hline I Promessi Sposi \\
$(1840)$
\end{tabular}

I numeri fra parentesi di nuovo si riferiscono ai casi in cui la proposizione col condizionale temporale viene condizionata da una protasi apparente.

Gli esempi del tipo: "Il guardiano rispose premurosamente con una frase di gesti: la prima parte della quale significava che la Signora non avrebbe mai bisogno di nessuno, e la seconda che $i$ padri avrebbero tenuta a guadagno ogni occasione di far cosa grata alla Signora.« ( $a$, II, I, p. 156), in cui il condizionale composto segue il semplice, sono di poca importanza, come c'insegna la tabella. Molto più istruttivi sono quelli in cui il composto viene seguito dal semplice, e $\mathbf{i}$ quali ammontano a dieci in tutte le redazioni. Ci limitiamo a citare solo un esempio di questo tipo, mostrando nello stesso tempo certi leggeri ritocchi che fece il Manzoni di redazione in redazione. Nella prima troviamo il periodo: "il guardiano gl'intimò che andrebbe a fare il noviziato a Modena, e che partirebbe all'indomani." ( $a$, I, IV, p. 68-69). Nella seconda, questo periodo viene un po' modificato, pur conservando due condizionali semplici: ,il guardiano gl'intimò che andrebbe a fare il suo noviziato a***, sessanta miglia lontano, e che partirebbe all'indomani.« (b; IV, p. 64). Ja redazione definitiva ci presenta un'ulteriore tendenza stilistica: "il guardiano gl'intimò che sarebbe andato a fare il noviziato a ${ }^{* * ;}$, sessanta miglia lontano, $e$ che partirebbe all'indomani." (c, IV, p. 66).

In questa applicazione parallela dei due condizionali sono incline a non vedere nessuna differenza temporale. Vorrei render più chiaro questo fenomeno esprimendolo con la formula: $q(a+b)$, dove in $q$ vedo l'elemento d'enfasi, cioè l'ausiliare sarebbe, che non si riferisce solo al participio che

coltello appuntato ed una fune, ed o vivi o morti pensasse di legarli; ed egli e ' $i$ figliuolo e tirerebbono su la fune del pozzo, alla quale accomandasse $l i$ detti porci.« (Le novelle, Firenze, 1888, LXX). 
precede (andato - segnato $a$ ), ma anche al condizionale semplice (partirebbe - segnato $b$ ) che gli succede.

Dopo aver esposto statisticamente l'uso del condizionale temporale nel romanzo manzoniano, discutendolo quanto ci permette lo spazio limitato, dobbiamo fermarci ad alcuni esempi che ci offrono un uso poco conosciuto di questa forma verbale. Forse il periodo: "Suppose ... che il partito potrebbe parer molto buono anche a lui; « (b, XXIX, p. 503; $c$, XXIX, p. 507) non attira la nostra attenzione, sebbene in questo caso possa parere più indicato un congiuntivo invece di "potrebbe«. Ma non di equivalenza fra il condizionale modale $e$ il congiuntivo vorremmo parlare ${ }^{10}$ in questo luogo, in cui più c'interessa osservare un'altra applicazione del condizionale. Pur potendo esser sostituito col congiuntivo - il cui uso riassume -, questa forma verbale è conservata, avenđo però la funzione del futuro in relazione al passato: »,aveva stipulato che il Cordova leverebbe l'assedio di Casale; ( $b$, XXVIII, p. 495), il che si ripete leggermente modificato nella definitiva: ",aveva stipulato che il Cordova leverebbe l'assedio da Casale; " (c, XXVIII, p. 491). Un altro esempio, tanto più eloquente che si trova in tutte tre le redazioni, ci avverte che non si tratta di nessuna trascuratezza da parte dell'autore, benché le costruzioni di questo tipo siano da trovare nel francese moderno:" "impose come condizione che l'uccisore di suo fratello partirebbe tosto da Cremona." ( $a$, I, IV, p. 68); "impose come una condizione, che l'uccisore di suo fratello partirebbe tosto di quella città.« ( $b, \mathrm{IV}, \mathrm{p} .63)$; "Finalmente richiese, impose come una condizione che l'uccisore di suo fratello partirebbe subito da quella città.. (c, IV, p. 65).

Se questa funzione del conđizionale, finora non registrata da parte dei grammatici, sia d'origine autoctona, cioè dialettale, o d'origine francese, dobbiamo ancora indagare prima di deciderci a dare una risposta definitiva. Però, in ogni caso è impossibile escludere l'influenza della lingua francese. se vediamo il seguente periodo fráncese del Goldoni: »;ils avoient arrêté dans. leurs assemblées, que toute fille qui en (des étudiants) recevroit chez elle, ne seroit jamais demandée en mariage par un citoyen de la ville, (Mémoires, I, 13), ${ }^{12}$ che due traduttori italiani ripetono in due maniere diverse. Uno sosti-.

${ }^{10}$ Quanto alla sostituzione del condizionale col congiuntivo e viceversa nell'italiano, v. G. Bertoni, Profilo linguistico d'Italia, Modena, 1940, p. 83. - Parlando. dello stesso problema nell'antico francése, L. Foulet, Petite syntaxe de l'ancien français, Paris, 1919, p. 143, dice: "Au moyen âge le subjonctif et le conditionel se tiennent de si près qu'il est légitime d'y voir deux aspects dífférents d'un mêmemode. Ils s'opposent tous deux de façon presque analogue à l'indicatif: l'indicatif est le mode de l'affirmation positive, les deux autres constituent le mode de l'affirmation conditionnelle ou de doute.. $\mathrm{Ma}$ subito aggiunge nella nota che segue in calce alla pagina che la sua affermazione si limita soltanto al condizionale-modo.

"Avviamo a certi periodi di questo tipo che si trovano copo il verbo wordonner巛: "Olaf, roi de Norvègé... ordonna que leurs. assemblées ne se tiendraient nulle autre part que... $\mathrm{H}$. Sten, Les temps du verbe fini (indicatif) en français moderne, Koebenhavn, 1952, pp. 71-72 e 87-88).

${ }^{12}$ Citato da: Carlo Goldoni, Mémoires, in Tutte le opere di Carlo Goldoni. a cura di Giuseppe Ortolani, tomo I, Milano, 1935. 
tuisce il condizionale francese col congiuntivo: "Essi avevano decretato nelle loro assemblee, che qualunque zitella avesse ricevuto in casa scolari, non fosse chiesta da verun cittadino,";' l'altro, invece, traspone la forma trovata nell'originale francese: "Avevano stabilito nelle loro adunanze che ogni ragazza che ricevesse studenti in casa non sarebbe mai stata chiesta in matrimonio da un cittadino di Pavia; $\aleph^{14}$.

\section{REZIM $\mathrm{E}$}

Temporalni kondicional u tri redakcije Manconijeva rom an a

Polazeći od činjenice da italijanski, za razliku od drugih zapadnoromanskih jezika, poseduje dva kondicionala-tempusa, autor ih analizira $u$ tri redakcije Manconijeva romana. Smatrajući da medju dva kondicionala $u$ ovoj primeni ne postoji nikakva temporalna razlika, on ih naziva "kondicional prosti« $i$ »kondicional složeni» umesto uobičajenog "kondicional sadašnji» i "kondicional prošli», a svoje tvrđenje dokazuje na nekim primerima iz Manconija u kojima se oba kondicionala javljaju naporedo $u$ istom periodu $u$ službi futura prošlosti.

Osvrćući se na upotrebu kondicionala-tempusa kod Manconija, on je pobrojao sve slučajeve njihove primene i prikazao ih statistički i procentualno. Na osnovu tananih tabela kao $i$ ispitivanja stanja $u$ pogledu iskazivanja posteriornosti $u$ odnosu na prošlost u svim epohama italijanskog jezika, dolazi se do zaključka da je Manconi u prvoj redakciji svog romana dao mnogo mesta kondicionalu složenom, dakle obliku koji je već u njegovo vreme služio kao skoro jedino živo sredstvo za iskazivanje ovog odnoša. U drugoj redakciji broj kondicionala prostih je jako povećan na račun složenih. Dalje, autor tvrdi da se u definitivnoj redakciji, koja je inače samo delimična izmena prethodne, Manconi naročito zadržao na kondicionalu. tempusu, umnogome povećavajući broj kondicionala složenih, ali da je pri svemu, ostajući na literarnoj tradiciji, uglavnom odstupio od normi koje je u ovom pogledu nalagao živi .jezik njegovih savremenika.

Naposletku, autor se zadržava na nekim slučajevima u kojima je kondicionalmodus nerazdvojan od konjunktiva kao i na primerima u kojima je kondicionalmodus preuzeo ulogu futura prošlosti.

${ }^{13}$ Citato da: Carlo Goldoni, Memorie, Milano, Sonzogno, sine anno.

${ }^{14}$ Citato da: Carlo Goldoni, Memorie, Milano, Vallardi, sine anno. 